DOCTRINA

\title{
El problema del concepto actual de establecimiento permanente en los convenios de doble tributación internacional frente a los nuevos desafíos fiscales en la economía digital
}

\author{
The problem of the existing concept of permanent establishment contained \\ in international double taxation conventions facing the new tax challenges \\ in the digital economy
}

\author{
Antonio Faúndez Ugalde \\ Pontificia Universidad Católica de Valparaíso, Chile
}

\begin{abstract}
RESUMEN Los nuevos desafíos fiscales en la economía digital propuestos por la OCDE no vienen más que a confirmar la obsolescencia del concepto de establecimiento permanente que ofrece el artículo 5 de los Modelos de Convenio para evitar la doble tributación. Sin embargo, las propuestas destinadas a buscar una solución a dicha obsolescencia frente a la economía digital ocultan un conflicto más complejo para Chile, que se concentra en la forma en que la jurisprudencia nacional ha interpretado los alcances de la referida cláusula del establecimiento permanente. Así, el presente artículo entrega un análisis sistemático y técnico del entorno legal del concepto de establecimiento permanente desde la óptica de la economía digital, que profundiza sobre aspectos que puedan mejorar su aplicación en casos concretos.
\end{abstract}

PALABRAS CLAVE Establecimiento permanente, doble tributación, BEPS, economía digital.

ABSTRACT The new tax challenges posed by the digital economy, as suggested by the OECD, confirm the obsolescence of the concept of permanent establishment offered by article 5 of the Model Convention to avoid double taxation. Nevertheless, the proposals aimed at finding a solution to such obsolescence on the digital economy conceal a more complex conflict for Chile, which focuses on the way Chilean jurisprudence has interpreted the scope of the permanent establishment's aforementioned clause. Thus, this article provides systematic and technical analysis of the legal environment of the concept of permanent establishment from the perspective of digital economy, deepening on aspects that can improve their application in specific cases. 
KEYWORDS Permanent establishment, double taxation, BEPS, digital economy.

\section{Introducción}

Las nuevas tecnologías han posibilitado a las empresas multinacionales generar inéditos modelos de negocios a través de operaciones desmaterializadas, como prestaciones de servicios o venta de bienes de contenido digital. Esto ha llevado a problemas de tributación directa e indirecta para los Estados frente a planificaciones fiscales agresivas que pueden derivar de vacíos legales, lo que ha trasladado los beneficios a países de baja o nula tributación. Es así como, en la reunión de San Petersburgo en septiembre de 2013, los países miembros de la OCDE y del G2O acordaron llevar a cabo un proyecto de trabajo conjunto destinado a eliminar la erosión de la base imponible y el traslado de beneficios (BEPS, por sus siglas en inglés), lo que fue materializado a través de la identificación de quince áreas estratégicas, denominadas "plan de acción BEPS». La primera acción, precisamente, está referida a cómo abordar los desafíos fiscales en la economía digital.

El primer informe, ${ }^{1}$ concluido en 2014, estableció que la economía digital se está convirtiendo cada vez más en la economía misma, por lo que sería difícil —si no imposible - separar a efectos fiscales la economía digital de la restante (OCDE, 2014: 8). Por lo tanto, a medida que la economía integra la tecnología digital, resulta cada vez más difícil segmentar la economía digital, que pasa a ser la economía propiamente dicha. Así, el informe de 2014 concluyó que para afrontar los desafíos fiscales y los problemas BEPS planteados por la economía digital, los esfuerzos deben enfocarse en analizar tanto las estructuras adoptadas por las empresas multinacionales como los nuevos modelos de negocios que la han asumido, entre los que se encuentran el comercio electrónico, las tiendas de aplicaciones, la publicidad en línea, la computación en la nube, plataformas participativas en red, la negociación de alta frecuencia y los servicios de pago en línea (OCDE, 2014: 82).

El mismo informe pone énfasis en la elusión artificial del estatus de establecimiento permanente del artículo 5 del Modelo de Convenio OCDE y la posibilidad de revisar dicho concepto, lo que fue ratificado en los informes del año 2015 (OCDE, 2015a: 10). Dentro de las principales observaciones se indicó que hoy es posible estar muy involucrado en la vida económica de otro país sin tener un lugar fijo de negocios o sin la necesidad de que esté presente un agente dependiente para celebrar un contrato, por lo que surge la preocupación de si la definición existente de establecimiento

1. Este primer informe estuvo a cargo del Grupo de Expertos sobre Fiscalidad de la Economía Digital (GEFED), órgano auxiliar del Comité de Asuntos Fiscales de la OCDE, en el que participan países no pertenecientes a la OCDE ni al G2o, en su calidad de asociados en igualdad de condiciones que los países miembros. 
permanente sigue siendo coherente con los principios subyacentes en los que se basa (OCDE, 2015b: 101).

Los últimos reportes de la OCDE al año 2017 dan cuenta de que las tecnologías de la información y la comunicación están incidiendo en la ciencia, la innovación, la economía y la forma en que las personas viven y trabajan, y que entre las más importantes tecnologías figuran los servicios informáticos en la nube (cloud computing), el internet de las cosas, la inteligencia artificial y la analítica de datos masivos (OCDE, 2017: 13). ${ }^{2}$ Esto impacta directamente en las operaciones transfronterizas, lo que dificulta la aplicación de la cláusula del establecimiento permanente del artículo 5 frente a las características propias de la economía digital, lo que se constituye en objeto de este trabajo. Para ello, será esencial considerar los Comentarios al Modelo de Convenio, cuya aplicación en Chile ha sido reconocida por la autoridad fiscalizadora, el Servicio de Impuestos Internos. Asimismo, en una dimensión dogmática normativa (Alexy, 1993: 29-34), se analizará de manera crítica la praxis jurídica de los tribunales de mayor jerarquía en Chile, de modo de establecer el alcance que han otorgado sus sentencias al concepto de establecimiento permanente.

\section{La cláusula del establecimiento permanente}

La legislación chilena no contempla una definición de establecimiento permanente, solo se limita a indicar en el número 1 del artículo 58 de la Ley sobre Impuesto a la Renta (Decreto Ley 824 de 1974) algunas situaciones que lo configurarían, como sucursales, oficinas, agentes o representantes. Sin embargo, las situaciones anteriores fueron incorporadas a la ley tomando en consideración los conceptos propios del derecho internacional. Si nos remontamos a la anterior ley del año 1964 (Ley 15.564), las comisiones unidas del Senado que discutieron sobre su texto señalaron lo siguiente:

Los tratados comerciales bilaterales emplean usualmente, como definición de establecimiento permanente, el lugar fijo de los negocios en que las actividades de

2. McLure (2000: 2-17) precisa que la comunicación es para la información lo que el transporte es para los bienes materiales: el modo de moverse a través del espacio, pues sin comunicación no puede haber comercio en el campo de la información. En tal sentido, sintetiza cuatro fases históricas: una primera fase antes de ser inventada la imprenta, en que el almacenamiento y la transmisión de las informaciones se realizaba manualmente o personalmente por los mismos individuos que generaban las obras (escultores, pintores, escribas, etcétera); segundo, la fase de la impresión, que permitió el almacenamiento tipográfico de las informaciones y su transmisión en forma impresa (libros, revistas, periódicos, etcétera); tercero, la era eléctrica analógica, que abrió camino a la transmisión electrónica de las informaciones y a la grabación, por parte del receptor, de las informaciones comunicadas electrónicamente; y cuarto, la era digital, que brindó la posibilidad de transmitir, a bajo costo, con facilidad y en forma rápida, informaciones almacenadas electrónicamente. 
las empresas se desarrollan, por entero o parcialmente, y cuyo giro sea cualquiera explotación de carácter remunerativo (Pérez, Pérez y Parga, 1966: 244).

Este es un claro indicio de que la idea de establecimiento permanente tuvo su origen en antecedentes internacionales que fueron recogidos por la legislación interna, para lo cual resalta como elemento esencial el lugar fijo de negocios, alcance que, como se verá, tuvo variaciones con posterioridad a la ley de 1964.

A partir de la segunda mitad del siglo XIX se comienza a hablar de esta forma de organización empresarial (Skaar, 1991: 83). En su evolución en el derecho prusiano se usó el término de betriebstatt para dilucidar la sujeción a la imposición de las utilidades derivadas de la actividad industrial y comercial de sujetos que vivían en otros municipios, lo que lleva a concluir que el origen del establecimiento permanente está en los impuestos sobre el producto y no en el impuesto sobre la renta (Montaño, 2004: 6). Así, por el año 1885, las leyes tributarias de Prusia recogieron el concepto de establecimiento permanente, que comprendía las agencias, los lugares de dirección, las sucursales y los lugares de venta de bienes. Posteriormente, en el año 1899, se incluyó por primera vez en un tratado internacional suscrito por el Imperio austrohúngaro y Prusia, lo que por cierto no significó su recepción de manera general (Montaño, 2004: 7). La definición que se daba en dicho tratado era la siguiente:

El término establecimiento comercial incluye sucursales, fábricas, depósitos, oficinas, lugares donde se realizan compras y ventas y otras instalaciones comerciales mediante las cuales el propietario, socio, gerente u otro representante permanente lleva a cabo sus actividades comerciales normales.

Con la creación de la Sociedad de Naciones por el Tratado de Versalles el 28 de junio de 1919, se estableció el primer Modelo de Convenio en el año 1927, el cual recogió la figura del establecimiento permanente, pero sin llegar a definirlo. Su regulación tomó como fundamento al principio de la fuente como una modalidad de pertenencia económica (economic allegiance). Sin embargo, la siguiente complejidad aparecía a la hora de determinar y asignar el rendimiento al establecimiento permanente, cuya solución más eficaz fue la utilización del criterio de la contabilidad separada, sin perjuicio de que el Modelo de Convenio hacía alusión al acuerdo necesario entre Estados contratantes para establecer las reglas de imputación de rentas, normalmente conforme con los rendimientos generados en cada uno de los territorios (Serrano, 2005: 151). Se sostiene que, por lo menos desde 1930, el consenso internacional ha sido que las utilidades deberían ser atribuidas a un establecimiento permanente basándose en el concepto de la empresa separada y la aplicación del principio arm's length (Baker y Collier, 2006: 26; García, 2005: 38).

Los estudios de la Sociedad de Naciones sirvieron de base para la elaboración de nuevos trabajos, entre los que se encuentra el Proyecto de Convenio de Doble Im- 
posición sobre la Renta y el Patrimonio del año 1963, elaborado por la OCDE. Este último modelo, en su artículo 5, planteó la siguiente definición de establecimiento permanente: «A efectos del presente convenio, la expresión "establecimiento permanente" significa un lugar fijo de negocios en el que una empresa realiza toda o parte de su actividad». Precisamente, esta definición fue la que se consideró en las comisiones unidas del Senado chileno que discutieron sobre el texto de la ley del año 1964.

En 1977 se elaboró un nuevo Modelo de Convenio de la OCDE — revisado en 2003-, el que introdujo una importante modificación al concepto de establecimiento permanente, en el siguiente tenor: «A efectos del presente convenio, la expresión "establecimiento permanente" significa un lugar fijo de negocios mediante el cual una empresa realiza toda o parte de su actividad». Con la primera redacción del año 1963 se quiso destacar más la función del lugar fijo de negocios en el que se realiza la actividad, mientras que, en la segunda redacción, el lugar fijo de negocios pasa a segundo plano para incidir en la realización de una actividad de la empresa a través del mismo (García, 1996: 95).

Lo anterior permite establecer un primer alcance sobre el verdadero sentido del enunciado «lugar fijo de negocios»: debería considerarse una interpretación amplia del concepto, la que permitiría incluso tener presentes las nuevas realidades de la actividad económica, en las que en algunos casos se llega a prescindir de las personas como medio para desarrollar una actividad empresarial (Faúndez, 2014: 14), como ocurre actualmente con los nuevos modelos de negocios que han asumido las empresas multinacionales en la economía digital, lo que será analizado más adelante. Previamente, se abordará el tratamiento internacional que ha tenido el referido enunciado, «lugar fijo de negocios».

\section{El lugar fijo de negocios}

Considerando una interpretación amplia de la cláusula del establecimiento permanente del artículo 5 del Modelo de Convenio de la OCDE, en su determinación debe primar la actividad empresarial propiamente tal que desarrolla una empresa en otro Estado. El párrafo 1 del artículo 3 del mismo Modelo de Convenio indica que el enunciado «actividad», relativo a una empresa, y el término «negocio» incluyen la prestación de servicios profesionales, así como cualquier otra actividad de naturaleza independiente. En ese sentido, la actividad empresarial puede tener nociones formalmente dispares que presuponen un tríptico conformado por el empresario, la actividad económica organizada y el establecimiento, los pilares del concepto jurídico unitario de la misma (Montaño, 2004: 41). Otros vinculan la actividad empresarial con el carácter comercial o el modo de realizarse la misma, como ocurre en el derecho alemán (Skaar, 1991: 248). En Estados Unidos prima el criterio de la obtención de un beneficio (González, 1992: 46). El Modelo de Convenio de la OCDE ha seguido la opinión de que el 
carácter productivo no debe formar parte de la definición de establecimiento permanente, ya que se trata de una característica derivada del simple ejercicio de la actividad empresarial (Baker, 1994: 90; Serrano, 2005, 151; Vogel, 1991: 208).

Así, actividad empresarial se posiciona en un elemento de la esencia de lo que es objeto de gravamen, cuya relación con el enunciado «lugar de negocios» no implica limitar la función del primero.

Genéricamente, el enunciado «lugar de negocios» incorporado en el artículo 5 se ha orientado en su aplicación como un elemento de carácter material, por lo cual describe distintas situaciones, como tener a disposición un local u oficinas. El alcance que ofrecen los Comentarios al Modelo de Convenio es que dicho término hace referencia a cualquier local, instalación o medios materiales utilizados para las actividades de la empresa, sirvan o no exclusivamente a ese fin, al margen de que la empresa sea o no la propietaria. ${ }^{3}$ Esto denota un carácter flexible o elástico del concepto de establecimiento permanente (Carmona, 2012: 42).

Entonces, al considerar una interpretación amplia o flexible del enunciado «lugar de negocios», se ha señalado que no requiere que sea de propiedad de ni estar alquilado por la empresa, como tampoco es necesario que sea exclusivamente ocupado por la empresa, pues puede estar situado en las facilidades de otra; puede existir aun cuando no existan locales disponibles o no sean necesarios para los propósitos del negocio y simplemente tengan un cierto espacio a su disposición; y puede estar ocupado legal o ilegalmente (Rohatgi, 2008: 224; Vogel, 1991: 206; García, 1996: 109). Así, se ha sostenido que es suficiente que el negocio empresarial se desarrolle en un espacio territorial del otro Estado contratante y no necesariamente en cuatro paredes (Faúndez y Blanco, 2009: 39). Lo anterior se refleja en el establecimiento permanente generado por un «agente dependiente», caso en el cual una persona actúa por cuenta de la empresa, con facultades suficientes para concluir negocios en representación de ella y que no necesariamente requiere para desarrollar su actividad un local o una oficina (Rohatgi, 2008: 229). De esta manera, aunque el local o lugar tenga capacidad para albergar en su seno una actividad de carácter empresarial o un negocio, más que su capacidad se requiere su utilización efectiva para el desarrollo de la actividad empresarial (García, 1996: 109).

Se ha presentado el caso, a la luz del Convenio entre Alemania y el Reino Unido, en que una habitación de hotel - asignada a una empresa no residente para desarrollar una actividad de gestión por un periodo de veinte años- fue declarada como lugar de negocios, lo que permitió establecer la presencia de un establecimiento permanente (Serrano, 2005: 167); por lo tanto, la actividad empresarial podría ser ejercida fuera del lugar de negocios (Rohatgi, 2008: 223). De esta forma, un lugar donde

3. «Commentaries on the articles of the Model Tax Convention», Organización para la Cooperación y el Desarrollo Económicos, artículo 5, párrafo 5.1.4, disponible en http://bit.ly/2sWSNpQ. 
operen equipos automáticos puede constituir un establecimiento permanente, si el equipo es operado y mantenido - después del montaje inicial— por la misma empresa o por sus agentes dependientes, como es el caso de máquinas expendedoras o de jugo, centrales telefónicas, estaciones de gasolina con autoservicio, equipos para recibir o transmitir señales de radio, equipos de cómputo u otros (Rohatgi, 2008: 226).

Los Comentarios de la OCDE indican que una empresa que solamente arriende equipos no tendrá un establecimiento permanente donde se use el equipo por parte del arrendatario, aun si la empresa provee de personal para operarlos. Sin embargo, si las responsabilidades de este personal van más allá del mantenimiento y la operación del equipo bajo la dirección, control y responsabilidad del arrendatario, la empresa puede estar ejecutando actividades y, por tanto, tener un establecimiento permanente. ${ }^{4}$

La línea argumentativa anterior demuestra que una interpretación literal del concepto de establecimiento permanente, de acuerdo con el significado natural de las palabras, puede conducir a un error en su aplicación.

Otro aspecto por considerar en el concepto de establecimiento permanente es la «fijeza». Al respecto, surge la siguiente interrogante: ¿la fijeza en el lugar de negocios debe tener una duración o permanencia determinada? Si se asume una interpretación restringida, la definición de establecimiento permanente no incluyó una fijeza temporal - salvo en los casos en que expresamente lo señala, como obras de construcción, instalación o montaje, prestación de servicios profesionales o por una empresa-, sino solo una física, es decir, la fijeza es la vinculación entre la instalación del negocio con un punto geográfico determinado, lo que ha sido denominado base approach o base theory (Skaar, 1991: 126). Sin embargo, el factor de fijeza debe ser interpretado de manera flexible, en atención a las variadas fórmulas de establecimientos permanentes que se pueden presentar, como por ejemplo el caso noruego de Hotel Platform, según el cual se consideró establecimiento permanente, previo acuerdo mutuo con Suecia, a una plataforma flotante que servía de alojamiento a los trabajadores de una plataforma petrolífera marina, aunque no estuviera fijada al suelo sino anclada (Skaar, 1991: 139).

Si bien los Comentarios de la OCDE reconocen en la fijeza un factor de territorialidad, también consideran un aspecto de permanencia, pero con un carácter flexible. Dichos Comentarios señalan que el lugar de negocios debe ser fijo en términos de tiempo y lugar, un situs definido donde la actividad empresarial es desarrollada (es decir, la prueba del lugar) con cierto grado de permanencia (esto es, la prueba de permanencia). ${ }^{5}$ En este sentido, un lugar de negocios puede convertirse en un establecimiento permanente desde el principio si se mantiene por un periodo que

4. «Commentaries», artículo 5, párrafo 8.

5. «Commentaries», artículo 5, párrafos 5-5.4. 
lo califique como tal. ${ }^{6}$ Asimismo, un establecimiento permanente empieza a existir cuando la empresa comienza sus actividades preparatorias para llevar a cabo su negocio mediante un lugar fijo de negocios, y deja de existir cuando todas las acciones conectadas con su actividad principal, su derecho a utilizarlo para fines del negocio, han sido terminadas. Por lo tanto, una interrupción temporal de las operaciones no puede ser vista como un cierre. ${ }^{7}$

Con relación a lo anterior, García (1996: 116-118) señala que la permanencia o temporalidad es un elemento que no aparece entre los destacados por la definición general de establecimiento permanente, por lo que no existe en principio ninguna exigencia explícita de que las actividades empresariales llevadas a cabo en un determinado lugar de negocios se extiendan durante un plazo de tiempo establecido, a excepción de la regla relativa a los lugares de construcción y otros que el mismo artículo señala. Agrega que, en los supuestos de los Comentarios de la OCDE, la permanencia no deriva del carácter fijo del lugar de negocios, sino que se toma en consideración como elemento decisivo determinante de la vinculación al territorio sustituyendo a la fijeza; no interviene como consecuencia de la relación del lugar de negocios con un punto geográfico determinado, sino como causa.

Con los antecedentes anteriores se puede concluir que el factor de permanencia, como presupuesto de existencia de un establecimiento permanente, no se encuentra sujeto, estrictamente, a un carácter continuo en el desarrollo de la actividad empresarial. En este sentido, determinadas actividades desarrolladas en forma discontinuada en el tiempo pueden ser calificadas como establecimientos permanentes, es decir, las interrupciones meramente temporales no afectan el grado de permanencia; la misma situación se presenta cuando dicha actividad se encuentra orientada a ser desarrollada por un largo periodo de tiempo, aunque por distintos motivos se produzca un término anticipado (Faúndez y Blanco, 2009: 44). Se debe considerar que el lugar fijo de negocios puede ser determinado, en primera instancia, por el punto geográfico en el cual se desarrolla; ahora bien, en el caso de existir dudas en su calificación, se puede recurrir a otros criterios, entre los cuales se encuentra el factor de carácter temporal, con la finalidad de acreditar la vinculación de la actividad empresarial con el territorio en el que se desarrolla.

\section{Dos sentencias de tribunales chilenos}

Si bien Chile es miembro de la OCDE desde 2010 y mantiene 32 convenios vigentes destinados a evitar la doble imposición, ${ }^{8}$ la discusión sobre la aplicación de la cláu-

6. «Commentaries», artículo 5, párrafos 6-6.3.

7. «Commentaries», artículo 5, párrafo 11.

8. «Convenios tributarios internacionales», Servicio de Impuestos Internos, disponible en http://bit. $1 \mathrm{y} / 2 \mathrm{Mi} 8 \mathrm{csR}$. 
sula del establecimiento permanente ha sido muy frugal a nivel jurisprudencial. A la fecha se conocen tres sentencias en las que se ha discutido sobre la existencia de un establecimiento permanente, entre las cuales destaca la pronunciada por la Corte Suprema el 13 de enero de 2014, en el caso Pirotecnia Igual SA con Servicio de Impuestos Internos. ${ }^{9}$

En este caso, la decisión de la Corte fue negar la existencia de un establecimiento permanente con relación a una persona física domiciliada en Chile, quien contaba con mandato para representar a la sociedad española Pirotecnia con facultades para adjudicarse contratos, cerrar negocios, cobrar honorarios y remesarlos a España. Si bien en una primera parte de la sentencia la Corte cita la definición de establecimiento permanente que entrega el artículo 5 del Convenio entre Chile y España, en la sentencia abandona el texto de los Modelos de Convenios de la OCDE y los Comentarios a los mismos, y posiciona cada uno de los vocablos del enunciado «establecimiento permanente» según las acepciones del Diccionario de la Lengua Española. Lo anterior denota el planteamiento excesivamente generoso utilizado por la Corte, que se apartó de todos los criterios desarrollados por años a nivel internacional, incluso bajo el compromiso asumido por Chile como miembro de la OCDE.

Un argumento similar se presentó en el caso Sud Americana de Vapores SA con Servicio de Impuestos Internos, cuya sentencia dictaminó que

la ley no ha definido lo que debe entenderse por agencia o establecimiento permanente; sin embargo, de conformidad con el uso general de esos conceptos, no puede sino inferirse que se trata de bienes u objetos comprendidos en el patrimonio de una sola persona. ${ }^{10}$

Se aprecia, al igual que en el caso Pirotecnia, que no se consideraron las directrices de la OCDE ni de la doctrina internacional, pues limitó su fundamento en la aplicación del derecho interno y bajo el uso ordinario o general de las palabras.

Antes del ingreso de Chile a la OCDE, la autoridad administrativa interpretadora de las normas tributarias chilenas (el Servicio de Impuestos Internos) planteaba que el concepto de establecimiento permanente consagrado en el artículo 5 de un convenio tributario en particular que estuviese basado en el Modelo de Convenio de la OCDE debía interpretarse de acuerdo con los Comentarios, incluso si no ne-

9. Pirotecnia Igual SA con Servicio de Impuestos Internos, Corte Suprema, rol 2245-2013. Las otras dos sentencias corresponden a la pronunciada el 25 de agosto de 2010, carátula Compañía Sud Americana de Vapores SA con Servicio de Impuestos Internos, Corte de Apelaciones de Valparaíso, rol 2383-2009, y la sentencia del 15 de diciembre de 2010, carátula Compañía Sud Americana de Vapores SA con Servicio de Impuestos Internos, Corte de Apelaciones de Valparaíso, rol 2382-2009.

10. Sentencia de Compañía Sud Americana de Vapores SA con Servicio de Impuestos Internos, Corte de Apelaciones de Valparaíso, rol 2383-2009, 25 de agosto de 2010. 
cesariamente tenían relación con la definición de establecimiento permanente que contempla la legislación interna; ${ }^{11}$ además, precisa que si bien los Comentarios elaborados por la OCDE no son jurídicamente vinculantes para Chile, tienen una particular importancia para el desarrollo del derecho tributario internacional, dado que han sido redactados y acordados por expertos que representan a los gobiernos de los países miembros de dicho organismo internacional, por lo que refleja el consenso internacional sobre la materia y pueden servir como parte de los medios de interpretación de un convenio de acuerdo con lo señalado en el artículo 31 de la Convención de Viena sobre derecho de los tratados, esto es, que los tratados deben interpretarse de buena fe conforme al sentido corriente de sus términos en el contexto del mismo y teniendo en cuenta su objeto y fin, lo que significa que se debe considerar y evaluar la opinión y jurisprudencia de otros órganos con la finalidad de llegar, en la medida de lo posible, a una interpretación internacionalmente común. ${ }^{12}$

En países como Canadá e Inglaterra, las cortes han aceptado los Comentarios de la OCDE como guía para la interpretación de los convenios. Así ocurrió en el caso Cudd Pressure Control Inc con The Queen, en el que la Corte canadiense sostuvo que «los Comentarios de la OCDE, por lo tanto, pueden proveer alguna asistencia para discernir el contexto legal que anteceden a las convenciones de doble tributación en el derecho internacional» (Rohatgi, 2008: 104). El mismo criterio declaró la Corte inglesa en el caso Sun Life Assurance of Canada con Pearson: «Es común [...] que se haga referencia a los Comentarios como guía para la interpretación del tratado» (Rohatgi, 2008: 105).

La International Fiscal Association indicó que, aun no siendo legalmente vinculables para los Estados contratantes, pues no forman parte de los convenios de doble imposición firmados por los Estados, los Comentarios adquieren una fuerza considerable cuando las cláusulas de los convenios se conforman de acuerdo con los postulados del Modelo de Convenio (García, 1996: 92).

Por lo tanto, el estándar de razonabilidad para establecer si se está o no en presencia de un establecimiento permanente debería conducirnos a la aplicación de la doctrina internacional, en especial los criterios que se han sostenido durante años sobre el artículo 5 y los compromisos asumidos por los Estados miembros de dicho organismo internacional. Pero las sentencias en los casos Pirotecnia y Sud Americana parecen indicar justamente lo contrario, al menos al sustentar su decisión en el significado natural del enunciado establecimiento permanente, tras recurrir, en el primer caso, al Diccionario de Lengua Española, lo que deja de lado el concepto legal que ofrece el artículo 5 del Convenio entre Chile y España y sus respectivos alcances en el

11. «Oficio 2521-2007», Servicio de Impuestos Internos, disponible en http://bit.ly/2MgL8KW; «Oficio 2890-2005», Servicio de Impuestos Internos, disponible en http://bit.ly/2sOlt43.

12. «Oficio 2176-2009», Servicio de Impuestos Internos, disponible en http://bit.ly/2MiC8VU. 
seno de los Comentarios de la OCDE. Esta situación es más crítica si se consideran los nuevos desafíos asumidos por la OCDE frente a la economía digital, lo que podría generar una evidente colisión en la búsqueda de una interpretación internacional común.

En esencia, la cláusula del establecimiento permanente debe ser interpretada a la luz de su definición legal entregada en el artículo 5 del Modelo de Convenio, el que debería ser preferido por sobre el significado natural de las palabras. El párrafo 2 del artículo 3 del Modelo de Convenio señala que cualquier término o expresión no definida en el Convenio tendrá, a menos que de su contexto se infiera una interpretación diferente, el significado que en ese momento le atribuya la legislación del Estado que lo aplique. Por ello, en los casos Pirotecnia y Sud Americana debió haber primado el concepto legal del artículo 5 , lo que también está en plena armonía con las reglas de interpretación del artículo 20 del Código Civil chileno, esto es, cuando un término de la ley ofrece un significado natural y un significado legal, debe preferirse este último si la materia así lo exige; cuando dicho término presenta dos significados naturales, pero solo uno de ellos determinado por el uso general del término, debe estarse a este último; cuando tiene dos significados naturales determinados por el uso general del término, debe atenderse al obvio en el que fue empleado (Guzmán, 2011: 138-139).

\section{Una interpretación amplia del concepto de establecimiento permanente frente a la economía digital}

Como se analizó antes, asumir una interpretación restringida de la definición de establecimiento permanente que ofrece el artículo 5 del Modelo de Convenio pareciera ser un camino errado, lo que generaría dificultades frente a los nuevos modelos de negocios desarrollados por las empresas multinacionales sobre la base de tecnologías de la información y comunicación. Por ejemplo, en el caso del comercio desarrollado en forma electrónica, ${ }^{13}$ implica un intercambio de información comercial a través de redes de telecomunicación, en los que pueden confluir acuerdos sin la presencia física de personas, por lo que queda ligado el consentimiento por la intermediación de dos sistemas computacionales (Jijena y Jijena, 2008: 65). ${ }^{14}$ Así, en un escenario de

13. Para referirse a este enunciado se utilizan con frecuencia términos como comercio electrónico, comercio digitalizado, comercio virtual, e-commerce o e-business, entre otros. Sin embargo, en atención a la precisión terminológica que requiere toda ciencia jurídica (Bobbio, 1950: 200), si al término «comercio» se añade el adjetivo "electrónico» implicaría un cambio en las características propias del término, frente a lo cual lo correcto es el uso del adverbio en lugar del adjetivo, que subraya la manera en que se efectúa el comercio (Corabi, 200o: 45).

14. Se ha coincidido que en este tipo de transacciones comerciales existe un pedido que se efectúa electrónicamente y los bienes o servicios se entregan de modo tangible o electrónico (Sprague y Boyle, 2001: 23). Así, las tres principales categorías de comercio desarrollado en forma electrónica son el uso de 
elusión tributaria y asumiendo una interpretación restringida del concepto de establecimiento permanente, un agente dependiente podría materializar en otro Estado la actividad empresarial de su mandante a través de operaciones electrónicas sin siquiera haber recorrido físicamente su territorio, con lo cual evita configurar un establecimiento permanente. Es una realidad, entonces, que las operaciones comerciales que se desplazan en este océano virtual generan el principal problema de no contar con una normativa especial, lo que podría derivar en contradicciones entre los Estados si cada uno decide aplicar las reglas y principios del derecho interno.

A fines de los años ochenta, la OCDE, en colaboración con otros organismos internacionales (Owens, 2000: 34), elaboró un conjunto de condiciones marco para el comercio internacional desarrollado en forma electrónica, en las que se plasmaron varios principios destinados a lograr un consenso internacional en la tributación en este tipo de operaciones. Estos principios fueron retomados dentro de las conclusiones de la Conferencia Ministerial sobre Comercio Electrónico celebrada en 1998 en la ciudad de Ottawa y, luego, trasladados a los informes del GEFED de 2014 y 2015, que plantearon que los principios fiscales fundamentales de neutralidad, eficiencia, certeza y simplicidad, efectividad y justicia, flexibilidad y sostenibilidad deben posicionarse a la luz de la proporcionalidad de los cambios necesarios para poner fin a los problemas fiscales que surgen en el contexto del marco fiscal internacional existente (OCDE, 2014: 162).

En cuanto al principio de neutralidad, el GEFED señaló la importancia de examinar una por una las distintas opciones para garantizar que su adopción no se traducirá en una discriminación a favor o en detrimento de una forma específica de negocio frente a otra; agregó que contribuyentes en situaciones análogas deberían estar sujetos a niveles impositivos equivalentes para evitar introducir distorsiones en el mercado (OCDE, 2014: 162). ${ }^{15}$ Entendiendo la neutralidad en un sistema fiscal como aquella que no distorsiona las decisiones de los agentes económicos (Griffith, Hines y Sørensen, 2010: 925-926; Altamirano, 2000: 43), pareciera que el informe del GEFED se posiciona en esa línea; sin embargo, al relacionar el principio de neutralidad con la equidad tributaria, lleva a un grado de confusión que es importante aclarar.

Lo anterior no significa que en cumplimiento del principio de neutralidad se debe presentar igual carga impositiva para todo tipo de operaciones, sea digital o convencional. Así, el tratamiento tributario diferenciado de operaciones en una economía digital - como sugieren algunos autores (Irribarra, 2015: 260) - debe ser entendido como una forma de cumplir con dicha neutralidad, pero bajo la óptica de evitar distorsiones económicas, sistemas más simples y minimizar las oportunidades de elu-

los medios electrónicos para la venta de bienes materiales o comercio sin conexión, la venta de contenido digitalizado o comercio en línea, y el acceso a internet y a las telecomunicaciones.

15. Esta misma idea se repite en el informe final del año 2015 (OCDE, 2015b: 20). 
sión (Badenes, 2014: 51). Es por ello que el principio de neutralidad va aparentemente encaminado a expresar que la tributación debe ser un medio y no un fin: el medio legítimo para las colectividades públicas de dotarse de recursos para proporcionar a sus poblaciones los servicios públicos necesarios, en un ambiente económico, social, educativo, cultural y más (Glaize, 2000: 56). Si bien la neutralidad no siempre es deseable, a menudo es valiosa e invariablemente será un punto de referencia importante para evaluar el sistema tributario (Mirrlees y otros, 2012: 656).

De esta forma, un ordenamiento jurídico que presente vacíos legales en el tratamiento tributario para determinadas operaciones en la economía digital puede significar tratos discriminatorios que colisionen con el principio de neutralidad. Ejemplo claro de ello es el cuestionamiento a los alcances que la jurisprudencia chilena ha otorgado al concepto de establecimiento permanente - como se analizó en los casos Pirotecnia y Sud Americana-, lo que podría dar lugar a decisiones corporativas destinadas a triangular determinadas operaciones digitales en Chile de manera de beneficiarse con una carga tributaria menor, que tendría como consecuencia distorsiones económicas y maniobras de elusión tributaria.

Una solución propuesta por el GEFED fue sustituir el concepto existente de establecimiento permanente por un criterio basado en una presencia económica significativa, a fin de responder a la naturaleza cambiante de las relaciones con los clientes en la economía digital, sin dejar de apoyarse, en parte, en una presencia física (OCDE, 2014: 159). Dicha presencia económica significativa estaría enfocada a establecer relaciones con los clientes o usuarios durante al menos seis meses, acompañadas de una cierta presencia física en el país, de forma directa o por medio de un agente dependiente; la venta de bienes o servicios por medios que impliquen una cercanía con los clientes en el país, como un sitio web en el idioma local, un servicio de entrega por parte de los proveedores ubicados en la jurisdicción, servicios bancarios y otros varios ofrecidos por los proveedores, o suministro de bienes o servicios procedentes de proveedores ubicados en dicho país; y el suministro de bienes o servicios a los clientes radicados en un determinado país fruto de una actividad sistemática de recogida de datos o aportaciones de contenidos por parte de personas situadas en el país en cuestión.

El referido nexo propuesto por el GEFED, además, plantea una retención definitiva en el Estado de la fuente; y en el caso de consumidores, para evitar aplicar dicha retención se propone asumir la responsabilidad por parte de las instituciones financieras involucradas en los pagos.

El criterio de la presencia económica significativa lleva necesariamente a replantear el concepto de establecimiento permanente que consagra el artículo 5 del Modelo de Convenio. El enunciado «lugar fijo de negocios» pasa a segundo plano y cobra relevancia la actividad económica propiamente tal desarrollada por el contribuyente en otro Estado. Sin embargo, la presencia económica significativa puede revestir aspectos 
subjetivos de difícil determinación. Son múltiples las formas de concretar un procedimiento y transmisión digitalizada de información, pero establecer cuánto es significativo y cuánto no tendrá una complejidad mayor. A esto se debe sumar la circunstancia de que la localización efectiva de personas físicas, jurídicas y de equipos informáticos, la facilidad de utilización de seudónimos y sistemas de encriptación de información por los usuarios de la red, la existencia de nuevos paraísos fiscales y, por último, la propia normativa en materia de protección de datos personales dificultan la identificación de las personas que intervienen en estas operaciones y su ubicación geográfica exacta, a efectos fiscales (Durán, 2005: 735). Además, si bien es posible determinar las ventas totales, como también los ingresos o las rentas de un vendedor, sería imposible determinar el lugar donde se realizan algunas ventas (McLure, 2000: 114).

En Chile, si se considera la interpretación asumida en los casos Pirotecnia y Sud Americana, sería muy difícil reconocer el factor de la presencia económica significativa para determinar la existencia de un establecimiento permanente. El criterio declarado en las citadas sentencias fue formulado atendiendo al texto literal del artículo 5 del Modelo de Convenio, esto es, de acuerdo con el sentido natural de las palabras, con lo que se desatiende, como consecuencia, el sentido que deriva de su contexto.

En países como España, la administración tributaria ha llegado a concluir que se estaría en presencia de un establecimiento permanente en todos aquellos territorios donde la empresa realice efectivamente operaciones de comercio en forma electrónica, es decir, en el lugar de la residencia fiscal del adquirente de los bienes o el beneficiario de los servicios (Durán, 2005: 740). En ese mismo país se han utilizado sistemas de interceptación denominados sniffers, consistentes en un dispositivo hardware o software diseñado para interceptar y almacenar información. Dichos sistemas se han aplicado, previo mandamiento judicial, en denuncias por distribución no autorizada de programas, obras multimedia y bases de datos jurídicas a través de internet. Tras treinta días de intervención telemática se obtuvieron pruebas concluyentes, ya que en los mensajes transmitidos y copiados en disco duro se hallaron catálogos, pedidos, órdenes de transferencias de fondos y los propios programas distribuidos ilícitamente (Segarra, 2004: 1.119).

Otro problema que podría derivar del criterio de la presencia económica significativa es la determinación de la atribución de los beneficios a establecimientos permanentes, los que pueden ser objeto de gravamen impositivo en la jurisdicción bajo la cual se encuentran operando. Conforme a ello, dichos beneficios atribuidos se constituyen en la ganancia que se esperaría que el establecimiento permanente genere si se tratara de una empresa distinta e independiente (OCDE, 2015b: 27).

Si se revisa el enfoque tradicional que ha operado en la OCDE, al alero de la Authorised OCDE Approach del año 2010, ${ }^{16}$ las soluciones con relación a la atri-

16. Centre for Tax Policy and Administration, «2010 report on the attribution of profits to permanent 
bución del beneficio se han centrado en las cuentas separadas del establecimiento permanente. Ahora bien, si un establecimiento permanente está constituido por actividades de un agente dependiente, el país de acogida puede tener derechos impositivos relacionados con dos entidades jurídicas distintas, a saber, la empresa agente y el establecimiento permanente agente dependiente, lo que se conoce como el dual taxpayer approach, que finalmente conduce a un análisis de dos tipos de transacción (Petruzzi y Holzinger, 2017: 275). Lo anterior es sin perjuicio de la discusión doctrinaria relacionada con el single taxpayer approach, en virtud del cual no se puede atribuir un beneficio al establecimiento permanente agente dependiente si se determina que tal beneficio ha recaído en forma exclusiva en el agente, en atención a las funciones realizadas por este último, los activos utilizados y los riesgos asumidos (Petruzzi y Holzinger, 2017: 277; Dziurdź, 2014: 136-138).

En la esfera de la economía digital, los trabajos desarrollados por el GEFED señalaron que la atribución de los beneficios es una consideración clave en el desarrollo de un nexo basado en una presencia económica significativa, lo que implica la necesidad de hacer cambios en las reglas de atribución de beneficios que lleven a asegurar la paridad, en la medida de lo posible, entre las empresas con presencia física de aquéllas que no lo tienen (OCDE, 2015b: 111). Sin embargo, el mismo informe da cuenta de que todos estos posibles ajustes deberán ser sustanciales en las normas existentes para la asignación de beneficios dentro de una empresa multinacional que opera en varias jurisdicciones, al considerar el análisis de funciones, activos y riesgos de las empresas en cuestión. De acuerdo con lo anterior, un método basado en la distribución fraccional de los beneficios ${ }^{17}$ podría generar cargas impositivas muy diferentes entre un establecimiento permanente con presencia física de otro con presencia virtual, lo que no sería recomendable.

En resumen, los modelos de negocios que han desarrollado las empresas sobre la base de nuevas tecnologías han generado un problema de adaptación del concepto de establecimiento permanente que ofrece el artículo 5 del Modelo de Convenio de la OCDE, el que data del año 1977. La administración tributaria chilena ha percibido esta dificultad y se ha posicionado en una línea interpretativa amplia, como se desprende de la Circular 57 emitida el 7 de diciembre de 2017. En el mismo instructivo se hace presente que los elementos que permiten verificar la configuración de un establecimiento permanente atienden «a la realidad dinámica de las actividades

establishments», Organización para la Cooperación y el Desarrollo Económicos, 22 de julio de 2010, disponible en http://bit.ly/2JLKXps.

17. La implementación de un método basado en la distribución fraccional requiere tres pasos sucesivos: primero, la definición de la base imponible a dividir; segundo, la determinación de las claves de asignación a dividir de esa base impositiva; y tercero, la ponderación de estas claves de asignación (OCDE, 2015b: 112). 
económicas», lo que es coincidente con lo que ha planteado la doctrina chilena en la última década, esto es, que frente a las nuevas necesidades del mercado, al considerar especialmente el carácter dinámico de la economía y las relaciones de sus agentes, una definición apropiada de establecimiento permanente que reúna los elementos esenciales al momento de ser calificado como tal se presenta como una prolongación, en otro país, de la actividad empresarial de su casa central, que puede o no estar relacionada con un lugar fijo de operaciones (Faúndez y Blanco, 2009: 34); así, cobra relevancia la actividad empresarial desplegada en otro Estado, independiente de la presencia física.

\section{Conclusiones}

La hipótesis de trabajo se ha centrado en establecer si la definición de establecimiento permanente que ofrece el artículo 5 del Modelo de Convenio de la OCDE se ajusta o no a las características propias de la economía digital, al contrastar dicho análisis con la praxis jurídica de las últimas sentencias de los tribunales superiores chilenos. Conforme a ello, la primera parte del estudio permitió concluir que las modificaciones que experimentó el concepto de establecimiento permanente del Modelo de Convenio OCDE desde 1963 a 1977 otorgaron preeminencia a la actividad empresarial en un territorio determinado por sobre el lugar fijo de negocios. Sin embargo, si bien ambos enunciados se relacionan y complementan, el segundo no implica una limitante del primero.

En segundo lugar, la propuesta de una interpretación amplia del concepto de establecimiento permanente que se encuentre en concomitancia con las características propias de la economía digital colisiona con el criterio jurisprudencial asumido en las últimas sentencias de los tribunales superiores de justicia chilenos, los que han formulado una interpretación literal de acuerdo al uso natural de las palabras, que deja de lado la definición legal del artículo 5 de los convenios tributarios vigentes de Chile, basados en el Modelo de Convenio de la OCDE y sus respectivos Comentarios.

En tercer término, se coincide con la doctrina que plantea la posibilidad de tratos diferenciados (no discriminatorios) entre operaciones de comercio convencional de aquéllas que se realizan en una economía digital (Irribarra, 2015: 260), pero debe ser entendido desde una perspectiva destinada a cumplir con el principio de neutralidad, esto es, con la finalidad de evitar distorsiones económicas, formular sistemas más simples y minimizar las oportunidades de elusión. Es por ello que un ordenamiento jurídico que presente vacíos legales en el tratamiento tributario para determinadas operaciones en la economía digital puede significar tratos discriminatorios que colisionen con el principio de neutralidad.

Finalmente, el estudio ha pretendido demostrar que el criterio de la presencia económica significativa plasmado en los informes de los grupos de expertos de la OCDE 
durante 2014 y 2015 lleva necesariamente a replantear el concepto de establecimiento permanente, pues pasa el enunciado del «lugar fijo de negocios» a un segundo plano. Sin embargo, la presencia económica significativa puede revestir aspectos subjetivos de difícil determinación, sobre todo al tomar en consideración las múltiples formas de concretar un procedimiento y transmisión digitalizada de información, lo que genera ambigüedad en valorizar lo significativo.

\section{Referencias}

Alexy, Robert (1993). Teoría de los derechos fundamentales. Madrid: Centro de Estudios Constitucionales.

Altamirano, Alejandro (2000). «La tributación directa ante el comercio electrónico y la fiscalización de las operaciones desarrolladas a través de internet». Ciência e Técnica Fiscal, 397: 7-67.

BAdenes, Nuria (2014). «Fiscalidad de las rentas del capital». En Opciones para una reforma del sistema tributario español (pp. 51-84). Madrid: Fundación Ramón Areces.

BAKER, Philip (1994). Double taxation conventions and international tax law. Londres: Sweet and Maxwell.

BAKer, Philip y Richard Collier (2006). "The attribution of profits to permanent establishments». En International Fiscal Association. Amersfoot: SDU Fiscale \& Financiële Uitgevers.

Bоввіо, Norberto (1950). Teoria della scienza giuridica. Turín: Giappichelli.

Carmona, Néstor (2012). «La noción de establecimiento permanente en los tribunales: Las estructuras operativas mediante filiales comisionistas». Crónica Tributaria, 145: 39-57.

Corabi, Giampaolo (2000). «Consecuencias tributarias del comercio electrónico». Criterios Tributarios, 14 (133): 43-56.

DURÁN, Vicente (2005). «Fiscalidad del comercio electrónico, especial referencia a la atribución de beneficios a establecimientos permanentes en este tipo de operaciones». En Fernando Serrano (compilador), Fiscalidad internacional. Madrid: Centro de Estudios Financieros.

DzIURDź, Kasper (2014). «Attribution of functions and profits to a dependent agent PE: Different arm's length principles under articles 7(2) and 9?». World Tax Journal, 2 (6): 135-167.

FAÚnDEZ, Antonio (2014). «Los establecimientos permanentes y el comercio internacional desarrollado en forma electrónica: ¿Una adecuación conceptual en los convenios de doble tributación internacional?», Curitiba, 4 (37): 6-33. Disponible en http://bit.ly/2JHobvZ. 
FaúndeZ, Antonio y María Blanco (2009). Establecimientos permanentes: Aspectos tributarios. Santiago: Librotecnia.

García, Francisco (1996). El establecimiento permanente, análisis jurídico tributario internacional de la imposición societaria. Madrid: Tecnos.

-. (2005). «Los precios de transferencia: Su tratamiento tributario desde una perspectiva europea». Crónica Tributaria, 117: 33-82.

Glaize, Antoine (2000). «La neutralidad y equidad tributaria en el comercio electrónico». En La tributación frente a las relaciones internacionales y la utilización de las nuevas tecnologías. Madrid: CIAT.

GonzÁLEZ, Victoriano (1992). «Instrucción del Inland Revenue Service para la aplicación del convenio de doble imposición entre España y los Estados Unidos». Tribuna Fiscal, 17: 39-57.

Griffith, Rachel, James Hines y Peter Sørensen (2010). «International capital taxation». Dimensions of tax desing (pp. 914-996). Oxford: Oxford University Press.

Guzmán, Alejandro (2011). Las reglas del Código Civil de Chile sobre interpretación de las leyes. Santiago: Legal Publishing.

IRRIBARRA, Eduardo (2015). «La equidad tributaria en el comercio digital según la OCDE, y sus desafíos para la recaudación fiscal». Revista de Estudios Tributarios, 14: 241-270. Disponible en http://bit.ly/2sZbkAQ.

Jijena, Hernán y Renato Jijena (2008). Comercio, facturas y factoring electrónico, análisis de la Ley 19.983. Santiago: Lexis Nexis.

McLure, Charles (2000). "Historia de la tecnología de las comunicaciones: Sus consecuencias en el campo tributario». En Giampaolo Corabi y Charles McLure (compiladores), La tributación sobre el comercio electrónico: Objetivos económicos, restricciones tecnológicas y legislación tributaria. Buenos Aires: Depalma.

Mirrlees, James, Stuart Adam, Timothy Besley, Richard Blundell y Stephen Bond (2012). «The Mirrlees review: A proposal for systematic tax reform». National Tax Journal, 3 (65): 655-684. DOI: 10.17310/ntj.2012.3.06.

Montaño, César (2004). Derecho tributario internacional, el establecimiento permanente. Bogotá: Temis.

OCDE, Organización para la Cooperación y el Desarrollo Económicos (2014). «Proyecto OCDE/G2o de erosión de bases imponibles y traslado de beneficios». Nota explicativa. Disponible en http://bit.ly/2Jw2LbS.

-. (2015a). «Proyecto OCDE/G2o de erosión de bases imponibles y traslado de beneficios». Nota explicativa. Disponible en http://bit.ly/2JUVQIG.

-. (2015b). Addressing the tax challenges of the digital economy: Action 1, 2015 final report. París: OECD Publishing. DOI: 10.1787/9789264241046-en.

-. (2017). OECD science, technology and industry scoreboard 2017: The digital trnasformation. París: OECD Publishing. DOI: 10.1787/9789264268821-en.

OwENs, Jeffrey (2000). «El comercio electrónico y la tributación». En La tributación 
frente a las relaciones internacionales y la utilización de las nuevas tecnologías. Madrid: CIAT.

Pérez, Lindor, Sergio Pérez y José PARga (1966). Reforma tributaria. Santiago: Jurídica.

Petruzzi, Raffaele y Raphael Holzinger (2017). «Profit attribution to dependent agent permanent establishments in a post-BEPS era». World Tax Journal, 9 (2): 263-300.

Rohatgi, Roy (2008). Basic international taxation. Londres: Legis.

Segarra, Santiago (2004). «Nuevas tecnologías y administración tributaria: Experiencia internacional y comunitaria». En Teodoro Cordón (compilador), Manual de fiscalidad internacional. Madrid: Instituto de Estudios Fiscales.

Serrano, Fernando (2005). «Los aspectos fiscales internacionales de los establecimientos permanentes». En Fernando Serrano (compilador), Fiscalidad internacional. Madrid: Centro de Estudios Financieros.

SkaAr, Arvid (1991). Permanent establishment erosion of a tax treaty principle. Deventer: Kluwer.

Sprague, Gary y Michel Boyle (2001). «Taxation of income derived from electronic commerce». Cahiers de Droit Fiscal International, 96 (1).

Vogel, Klaus (1991). On double taxation conventions. Deventer: Kluwer Law International.

\section{Sobre el autor}

Antonio Faúndez Ugalde es abogado. Licenciado en Ciencias Jurídicas por la Universidad Arturo Prat de Chile. Magíster en Gestión mención Tributación Internacional y doctor en Derecho, ambos por la Pontificia Universidad Católica de Valparaíso, Chile. Se desempeña como profesor investigador asociado de la Escuela de Comercio de la Pontificia Universidad Católica de Valparaíso. Su correo electrónico es antonio. faundez@pucv.cl. 


\title{
REVISTA CHILENA DE DERECHO Y TECNOLOGÍA
}

La Revista de Chilena de Derecho y Tecnología es una publicación académica semestral del Centro de Estudios en Derecho Informático de la Facultad de Derecho de la Universidad de Chile, que tiene por objeto difundir en la comunidad jurídica los elementos necesarios para analizar y comprender los alcances y efectos que el desarrollo tecnológico y cultural han producido en la sociedad, especialmente su impacto en la ciencia jurídica.

\author{
EDITOR GENERAL \\ Daniel Álvarez Valenzuela \\ (dalvarez@derecho.uchile.cl) \\ SITIO WEB \\ rchdt.uchile.cl \\ CORREO ELECTRÓNICO \\ rchdt@derecho.uchile.cl \\ LICENCIA DE ESTE ARTÍ́CULO \\ Creative Commons Atribución Compartir Igual 4.0 Internacional
}

\begin{abstract}
y
La edición de textos, el diseño editorial

y la conversión a formatos electrónicos de este artículo

estuvieron a cargo de Tipográfica

(www.tipografica.cl).
\end{abstract}

